\title{
Buchführung unter Verwendung der bereits registrierten Zahlungsverkehrsdaten
}

P. Schneider

Korrespondenz: Peter Schneider, dipl. Wirtschaftsprüfer FMH Services Treuhand Thorackerstrase 3 CH-3074 Muri bei Bern Tel. 0319518840 Fax 0319518839

E-Mail:

peter.schneider@fmhtreuhand.ch

\section{Einleitung}

Der Aufbau einer Finanzbuchhaltung hat sich über die Jahre nicht wesentlich verändert. Einzig die Kontenpläne wurden von Zeit zu Zeit überarbeitet und den Kundenbedürfnissen angepasst. Was sich verändert hat, sind die Einsatzmöglichkeiten von technischen Hilfsmitteln, wie Computer, Buchhaltungsprogramme usw. Aber auch die Organisation des Zahlungsverkehrs kann zu wesentlichen Vereinfachungen in bezug auf die Jahresabschlusserstellung führen.

Ausgehend von der klassischen Erstellung der Bilanz und Erfolgsrechnung durch das Führen der Grundbücher, Kontierung, Datenerfassung, Verarbeitung können bei Anwendung des elektronischen Zahlungsverkehrs die bereits erfassten Daten weiterverwendet werden. Wie sieht dies konkret aus?

\section{Varianten der Grundbuchführung, Kontierung, Datenerfassung, Verarbeitung}

- Die Grundbuchführung erfolgt durch den Kunden, sei es in speziell dafür entwickelten Büchern oder direkt auf dem jeweiligen Kontoauszug der Bank oder Post. Der Treuhänder kontiert, erfasst und verarbeitet die Daten und erstellt den Jahresabschluss.

- Die Grundbuchführung und Kontierung erfolgt durch den Kunden. Der Treuhänder erfasst und verarbeitet die Daten und erstellt den Jahresabschluss.

- Die Grundbuchführung, Kontierung und Datenerfassung erfolgt durch den Kunden. Voraussetzung ist der Kauf eines Finanzbuchhaltungsprogrammes. Die so erfassten Buchhaltungsdaten werden dem Treuhänder mittels Diskette zur Jahresabschlusserstellung zur Verfügung gestellt.

\section{Automatisierte Verarbeitung der registrierten Zahlungsverkehrsdaten}

Der Kunde erstellt seine Buchhaltung automatisiert im Anschluss an die Erfassung der Electronic-Banking-Daten. Setzt der Kunde seinen Computer ebenfalls für die Abwicklung seines Zahlungsverkehrs ein, so kann er bereits beim Erfassen des Telebankingauftrages die Kontierung vornehmen. Bei diesem Arbeitsgang wird gleichzeitig die Kontonummer eingegeben. Danach werden die erfassten Daten an die Bank zur Zahlung freigegeben. Zusätzlich werden in einem Datenpool Finanzbuchhaltung die gleichen Daten gespeichert und über ein Schnittstellenprogramm in die Finanzbuchhaltung eingespiesen.

Die Buchhaltung wird somit automatisch aufgebaut. Dies geschieht durch die vom System erstellten Buchungssätze. Die so verarbeiteten Buchhaltungsdaten werden dem Treuhänder mittels Diskette zur Jahresabschlusserstellung zur Verfügung gestellt.

\section{Unterschiede zur konventionellen Verarbeitung}

Die Arbeitsschritte des Kontierens der Grundbücher und das Erfassen am PC fällt weg. Der Kunde gelangt somit direkt zu den Daten für die Erstellung der provisorischen Bilanz und Erfolgsrechnung. Zu beachten gilt es jedoch, dass die automatisch erstellten Buchungen mit dem Grundbuch der Bank oder Post abgestimmt werden müssen. Der noch nicht registrierte Buchungsverkehr ist somit manuell noch zu ergänzen.

\section{Hilfsmittel}

Der Kunde wickelt den Zahlungsverkehr bereits elektronisch ab oder beabsichtigt, dies in naher Zukunft zu tun. Für die Verarbeitung der automatisch erstellten Buchungen benötigt der Kunde ein Finanzbuchhaltungsprogramm sowie ein Schnittstellenprogramm zwischen der E-Banking-Software und dem Finanzbuchhaltungsprogramm. 


\section{Ablaufschema}

a) konventionelle Variante

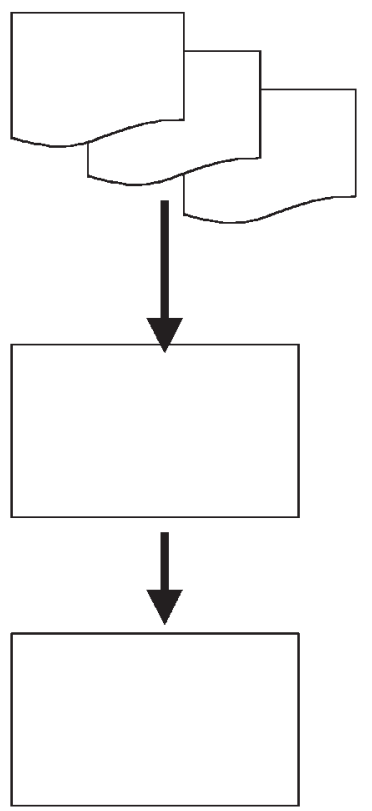

Grundbücher

- Kasse

- Postcheck

- Bank

Erfassen

Verbuchen

in Finanzbuchhaltung)

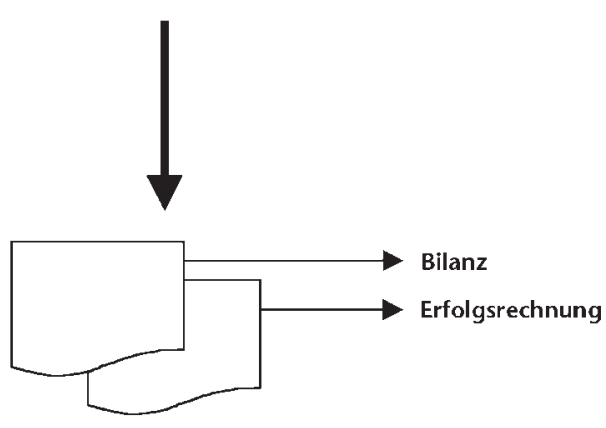

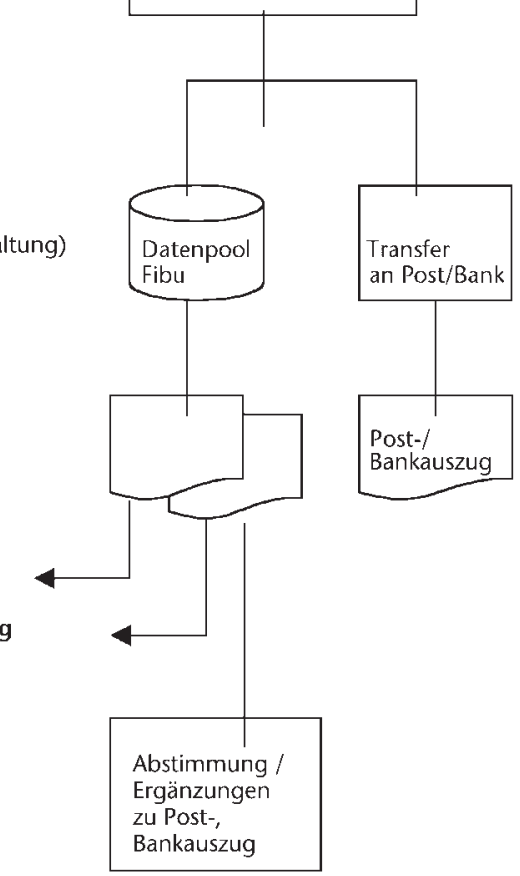

b) automatisierte Variante

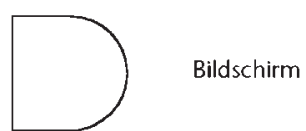

Erfassen

Kreditoren und

Konto-Nr.

Finanzbuchhaltung

\section{Zusammenfassung}

Der PC hat sich heute einen festen Platz in der Arztpraxis erobert. Für die Leistungserfassung und Fakturierung ist er zum unentbehrlichen Hilfsmittel geworden. Durch den erfolgreichen Einsatz des PCs in der Patientenadministration wächst das Interesse an einer erweiterten Nutzung der EDV-Infrastruktur, beispielsweise im Bereich der Praxisbuchführung. Ihr FMH-Services-Treuhänder hilft Ihnen gerne bei der Umsetzung Ihres Vorhabens.

www.FMHjob.ch

- Stellen- und Praxisvermittlung online

- Service en ligne de placement et de remise de cabinets

Für Fragen kontaktieren Sie unsere beiden Damen Frau Logovi und Frau Born von der Abteilung Stellenvermittlung in Bern unter Tel. 0313591212 oder E-Mail fmhstv@ hin.ch.

Si vous souhaitez obtenir de plus amples informations, n'hésitez pas à prendre contact avec Madame Logovi ou Madame Born de «l'Office de placement» à Berne au numéro de téléphone $031 \quad 359 \quad 12 \quad 12$ ou e-mail fmhstv@hin.ch.

\section{FMH Consulting Services}

Stellen- und Praxisvermittlung /

Office de Placement,

Elfenstrasse 18, 3000 Bern 16,

Tel./tél. 03135912 12, Fax/fax 03135911 12,

E-Mail:fmhstv@hin.ch
Unsere Treuhandspezialisten im deutschsprachigen Raum

Geschäftsstelle Ostermundigen Herr Harry Huwiler, Tel. 0319390139

Geschäftsstelle Olten

Herr Peter Senn,Tel. 0622059035

Geschäftsstelle Basel

Herr Linus Cavegn, Tel. 0613195121

Geschäftsstelle Lohn-Ammannsegg SO, Herr Rolf Lehmann, Tel. 0326775442

Geschäftsstelle Muri/AG

Herr Roland Bütler, Tel. 0566640309
Geschäftsstelle Cham

Herr Guido Schmid, Tel. 0417486290

Geschäftsstelle Sursee

Herr Patrik Dahinden, Tel. 0419267045

Geschäftsstelle Stans

Herr Marcel Helfenstein, Tel. 0416111821

Geschäftsstelle Zürich-Wiedikon

Herr Christoph Lautenschlager, Tel. 014571575

Geschäftsstelle Winterthur Herr Urs Gross, Tel. 0522240241

Geschäftsstelle Weinfelden

Herr Adrian Hartmann, Tel. 0716228686
Geschäftsstelle Oberuzwil/SG

Herr Martin Brenner, Tel. 0719513066

Geschäftsstelle Neuhausen a. Rheinfall Herr Peter Oechslin, Frau Katja Sigrist, Tel. 0526755925

Geschäftsstelle Speicher/SG Herr Jürg Schmid, Tel. 0713442175

Geschäftsstelle Au/SG

Herr Anibal Alghisi, Tel. 0717401787

Geschäftsstelle Chur

p.Adr. Riedi Ruffner Theus AG,

Tel. 0812584646 